\title{
Antibody Cross-Reactivity to Hemagglutinin Protein Antigens Demonstrates Feasibility for Development of a "Universal" Influenza A Synthetic Peptide Vaccine
}

\author{
Ziqing Jiang, Lajos Gera, Colin T. Mant, and Robert S. Hodges \\ Department of Biochemistry and Molecular Genetics, University of Colorado Denver, \\ Anschutz Medical Campus, School of Medicine, Aurora, CO, 80045, USA
}

\section{Introduction}

Influenza A viruses spread rapidly, causing widespread seasonal epidemics of respiratory disease worldwide, which results in more than a billion cases and 500,000 deaths annually [1]. Current influenza vaccines primarily elicit antibodies against the receptor-binding region of the head domain of the hemagglutinin (HA) glycoprotein trimer (Figure 1). This region is hyper-variable and highly mutable, leading to new forms of the virus that can evade neutralizing antibodies. The stem region of HA contains highly conserved $\alpha$-helical sequences as a result of their functional role in membrane fusion and virus entry. Studies have shown that a few rare neutralizing human monoclonal antibodies can recognize these highly conserved epitopes and neutralize both homotypic and heterotypic influenza strains $[2,3]$. There are some $18 \mathrm{HA}$ subtypes in influenza A virus that infect humans, animals and birds (Figure 1) [4].

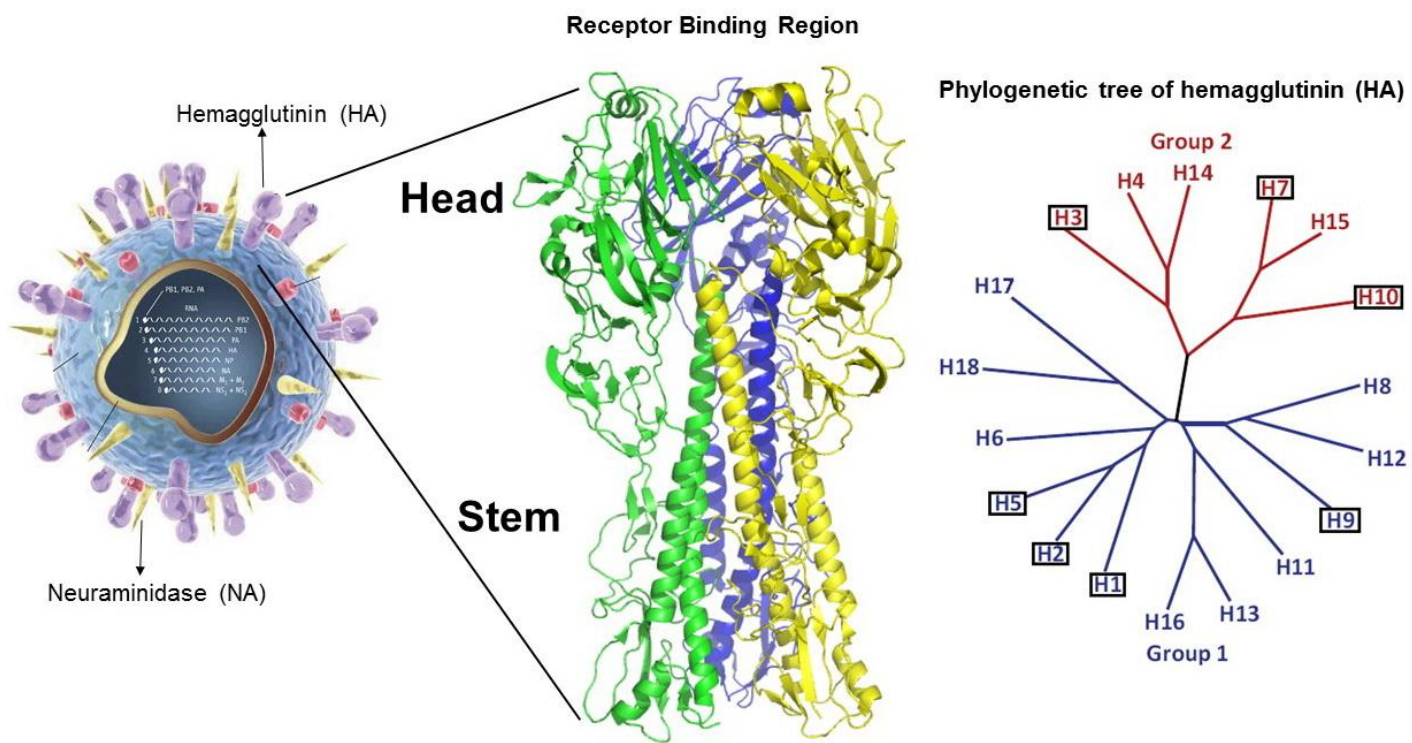

Fig. 1. The influenza virus with surface proteins hemagglutinin (HA) and neuraminidase (NA) is shown in the left panel. The middle panel shows the X-ray structure of the HA trimeric glycoprotein. The right panel shows the phylogenetic tree of HA, group 1 (colored blue) and group 2 (colored red). The subtypes that have been confirmed in humans are boxed.

Subtypes associated with pandemic human influenza are $\mathrm{H} 1, \mathrm{H} 2$ and $\mathrm{H} 3$. H1 and $\mathrm{H} 3$ subtypes are continually undergoing immune selection (antigenic drift), so that antibody-resistant strains continue to emerge and cause new human pandemics, necessitating formulation of new vaccines every year [5,6]. Subtypes H5, H7 and H9 are recognized by the World Health Organization as "potential pandemic viruses" and humans have no pre-existing immunity to these subtypes [7]. 
Our strategy is to develop a "Universal" Influenza Synthetic Peptide Vaccine based on highly conserved $\alpha$-helical epitopes in the stem region of HA. Such a vaccine would not have to be changed annually and would protect against antigenic drift as well as antigenic shift variants. Synthetic peptide vaccines have major advantages over existing vaccine approaches to prevent viral infections when there is a detailed understanding of the mechanism of virus entry into the cell: 1) one can select the target protein and a particular sequence within the target protein; 2) side-effects from unnecessary proteins, other molecules and unnecessary epitopes within the target protein are eliminated; and 3) simplicity, small size, easy to manufacture and the low cost of goods.

\section{Results and Discussion}

Our platform technology consists of a disulfide-bridged two-stranded $\alpha$-helical coiled-coil peptide template of 29 residues per strand where the $\alpha$-helical epitopes from the HA protein are inserted into the template to display the exposed helical surface of the native protein. The templated B-cell epitopes have five out of every seven residues exposed on the surface of the coiled-coil with the two-stranded

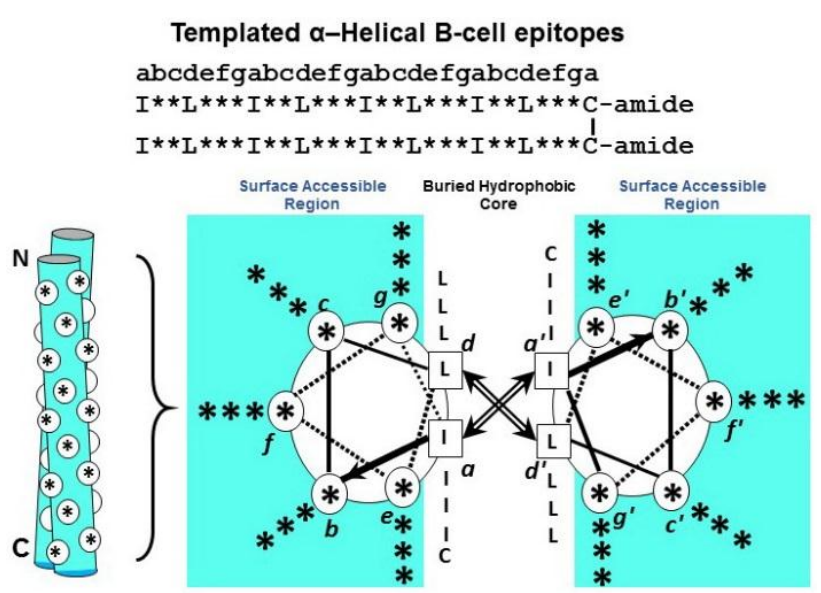
helical conformation stabilized by converting the native buried hydrophobic residues to Ile/Leu residues (Figure 2).

Fig. 2. A two-stranded $\alpha$-helical coiledcoil template to display $\alpha$-helical epitopes. In the cross-sectional view, the direction of the $\alpha$-helices is into the page from the $\mathrm{NH}_{2}$ to the $\mathrm{COOH}$ terminus, with the polypeptide chains parallel and in-register. In a homo-two-stranded coiled-coil two identical $\alpha$-helical B-cell epitopes are displayed. The templatestabilizing disulfide-bridge is also buried in the hydrophobic core between the two $\alpha$-helices. Antibodies generated to this immunogen bind the corresponding $\alpha$ helix in the native HA protein.

\section{Templated Peptide Immunogen with T-cell and B-cell Epitopes}

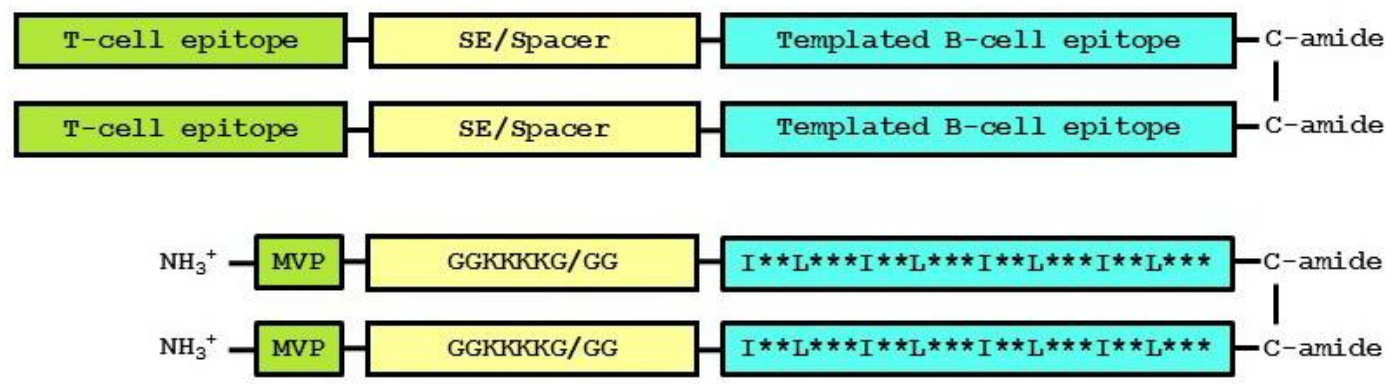

Fig. 3. Templated peptide immunogens where each polypeptide chain contains an eighteen residue $T$-cell epitope followed by a positively charged solubility enhancer (SE) (if required) or a two-Gly spacer, a 28-residue helical B-cell epitope from the protein of the interest and a C-terminal disulfidebridge to further stabilize the helical conformation. The T-cell epitope is denoted MVP for a measles virus promiscuous T-cell epitope [8]. 
The peptide immunogens used in this study are shown in Figure 3. Each immunogen consists of two polypeptide chains containing an $N$-terminal T-cell epitope, a solubility enhancer region or spacer, a 28-residues B-cell epitope and a $C$-terminal disulfide-bridge.

Shown in Figure 4 is the sequence of immunogen 4P1 (residues 401-428). The antibodies to this immunogen were generated in rabbits after three immunizations on days 1,15 and 44. IgG was purified from the final rabbit sera and used to evaluate antibody cross-reactivity to a series of H1N1 subtypes and seven different HA proteins from subtypes H1N1, H2N2 and H5N1 from group 1 and H3N2, H7N7 and H7N9 from group 2. The results indicate that the antibodies to immunogen 4P1 (401-428) cross-react with all H1N1 subtypes tested (Figure 4, panel A). As shown in the middle panel of Figure 4, the H1N1 sequences from 1934 to present are highly conserved in this 4P1 region which explains the excellent cross-reactivity of 4P1 antibodies to H1N1 subtypes (Figure 4, panel A). Though the 4P1 sequence has the potential to be a vaccine against all $\mathrm{H} 1 \mathrm{~N} 1$ subtypes, which would be a major advantage over existing vaccines, the antibodies are not significantly cross-reactive with H5N1 and H2N2 from group 1 and group 2 HA antigens H3N2, H7N7 and H7N9 (Figure 4, panel B). In Figure 4 panels A and B the negative control HA protein for ELISA was the Brisbane/59/07 from H1N1. This HA protein contained residues 18-343 and is missing the antibody binding site 401-428 used in our vaccine construct for raising polyclonal antibodies in rabbits. It is very clear why these antibodies do not bind to H2N2, H5N1, H3N2, H7N7 and H7N9. Non-conservative substitutions in these HA proteins are indicated in pink in the middle panel of Figure 4.
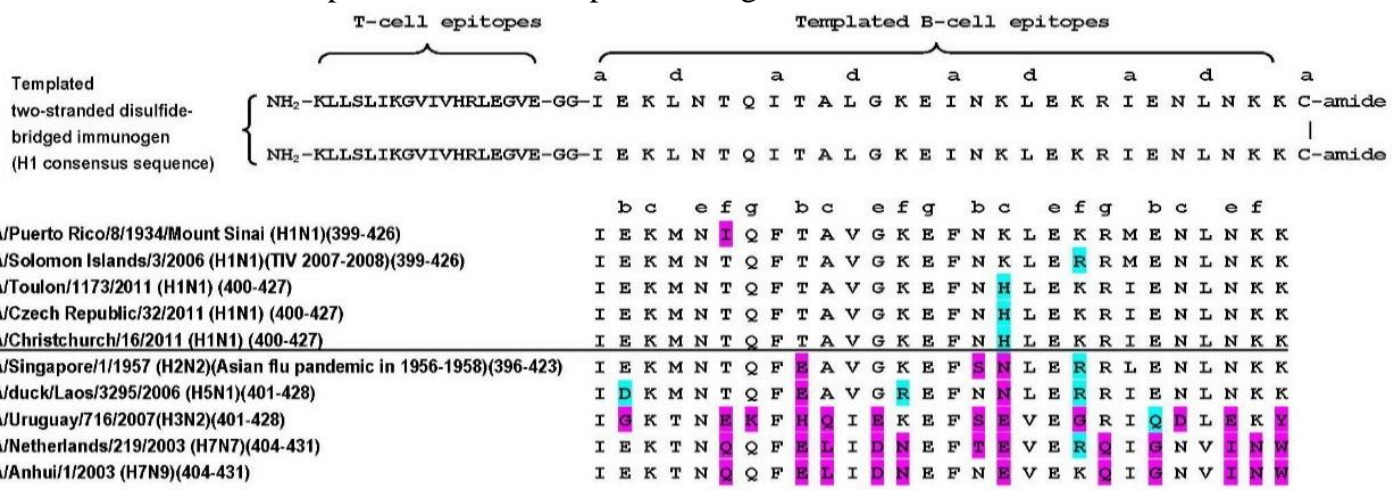

A/Puerto Rico/8/1934/Mount Sinai (H1N1)(399-426) A/Solomon Islands/3/2006 (H1N1)(TIV 2007-2008)(399-426) A/Toulon/1173/2011 (H1N1) (400-427) A/Czech Republic/32/2011 (H1N1) (400-427) A/Christchurch/16/2011 (H1N1) (400-427) A/Singapore/1/1957 (H2N2)(Asian flu pandemic in 1956-1958)(396-423) A/duck/Laos/3295/2006 (H5N1)(401-428) A/Uruguay/716/2007(H3N2)(401-428) A/Netheriands/219/2003 (H7N7)(404-431) A/Anhui/1/2003 (H7N9)(404-431)
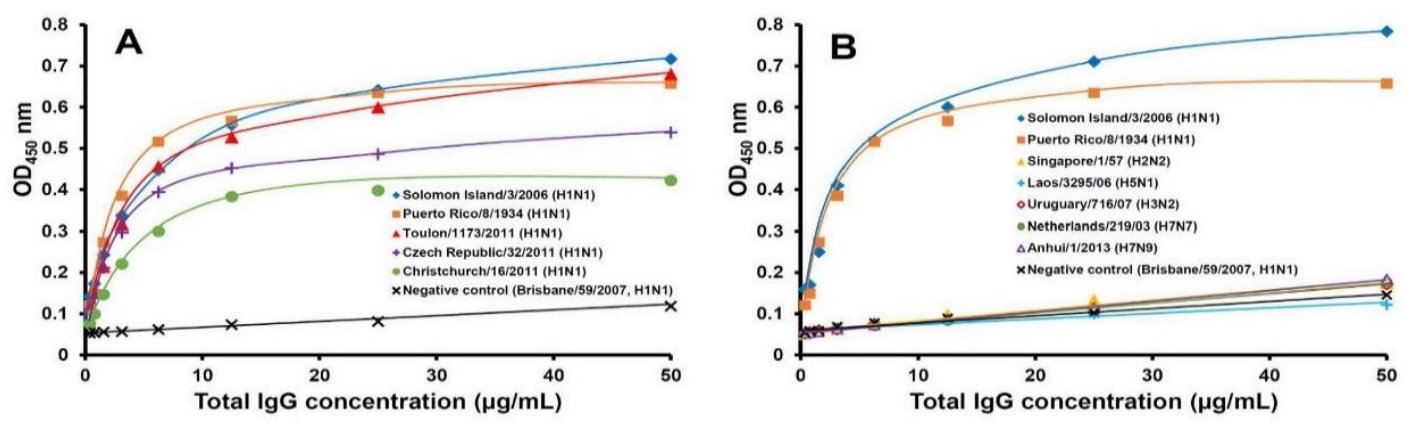

Fig. 4. Top panel shows the sequence of the synthetic peptide vaccine consisting of a HI consensus sequence, 4P1, inserted into the disulfide-bridged two-stranded $\alpha$-helical coiled-coil template stabilized by Ile and Leu residues at positions $\boldsymbol{a}$ and $\boldsymbol{d}$. The middle panel shows the sequences of five different H1N1 sequences and five representative sequences from H2N2, H5N1, H3N2, H7N7 and H7N9. Conservative substitutions are indicated in blue and non-conservative substitutions are indicated in pink. The lower panel shows the ELISA results of antibody binding to five different HA proteins from H1N1 and the negative control HA protein, Brisbane/59/07 (H1N1), which consists of amino acid residues 18-343 (panel A). Panel B shows the ELISA results of antibody binding to two HI and five HA proteins from H2N2, H5N1, H3N2, H7N7, H7N9 and the Brisbane negative control HA protein. 
To obtain cross-reactive antibodies to group 1 and group 2 HA proteins, work is in progress to use region 5P (421-449). This region was shown by our group to provide cross-reactive antibodies to group 1 and 2 when the synthetic disulfide-bridged two-stranded coiled-coil template was covalently linked to Keyhole Limpet Hemocyanin (KLH) [9].

What is most informative from these results is that an 18-residue promiscuous T-cell epitope from measles virus fusion $(\mathrm{F})$ protein $(\mathrm{MVF})$ can replace the need to conjugate the synthetic peptide immunogen to a carrier protein like keyhole limpet hemocyanin (KLH) as we did previously [9] and still generate an excellent antibody response. The T-cell epitope utilized in this study was first identified in 1990 [8] and first reported in synthetic peptide vaccine constructs in 1995 [10].

In conclusion, we first demonstrated the usefulness of our two-stranded $\alpha$-helical coiled-coil template technology in our studies to develop a synthetic peptide vaccine against Severe Acute Respiratory Syndrome (SARS) [11,12]. Here we have incorporated a 28-residue $\alpha$-helical sequence from the stem domain of hemagglutinin from $\mathrm{H} 1 \mathrm{~N} 1$ and showed that we can generate polyclonal antibodies that cross-react with all $\mathrm{H} 1 \mathrm{~N} 1$ subtypes in the region 401-428. These results suggest that a synthetic peptide vaccine for H1N1 including this region would not have to be changed annually and would protect against antigenic drift and antigenic shift variants. This technology has general applicability to be used to generate antibodies to any surface exposed $\alpha$-helical region in any protein.

\section{Acknowledgments}

We thank the John Stewart Endowed Chair in Peptide Chemistry to Robert S. Hodges for financial support. We are very grateful for the following reagents obtained through BEI Resources, NIAID, NIH: Hemagglutinin (HA) Proteins from Influenza Virus, Recombinants from Baculovirus, A/Solomon Islands/3/2006 (H1N1), NR-15170; A/Puerto Rico/8/1934 (H1N1), NR-19240; A/Toulon/1173/2011 (H1N1)pdm09, NR-34587; A/Czech Republic/32/2011 (H1N1)pdm09, NR-42486; A/Christchurch/16/2011 (H1N1)pdm09, NR-42487; A/Brisbane/59/2007 (H1N1), NR-13411; A/Singapore/1/1957 (H2N2), NR-2668; A/duck/Laos/3295/2006 (H5N1), NR-13509; A/Uruguay/716/2007 (H3N2), NR-15168; A/Netherlands/219/2003 (H7N7), NR-2633; A/Anhui/1/2013 (H7N9), NR-45118.

\section{References}

1. http://www.who.int/topics/influenza/en/

2. Sui, J., et al. Nat. Struct. Mol. Biol. 16, 265-273 (2009), http://dx.doi.org/10.1038/nsmb.1566

3. Wang T.T., Palese, P. Nat. Struct. Mol. Biol. 16, 233-234 (2009), http://dx.doi.org/10.1038/nsmb.1574

4. Wu, Y., Wu, Y., Tefsen, B., Shi, Y., Gao, G.F. Trends Microbiol. 4, 183-191 (2014), http://dx.doi.org/10.1016/j.tim.2014.01.010

5. Subbarao, K. et al. Immunity 24, 5-9 (2006), http://dx.doi.org/10.1016/j.immuni.2005.12.005

6. Doherty P.C., Kelso A. J. Clin. Invest. 118, 3273-3275 (2008), http://dx.doi.org/10.1172/JCI37232

7. Fiore, A.E., Bridges, C.B. and Cox, N.J. (2009) Seasonal Influenza Vaccines. In Vaccines for Pandemic Influenza. (Compans, R.W. and Orenstein, W.A., Eds.) (pp. 43-82). Springer Verlag, Berlin Heidelberg.

8. Partidos, C.D., Steward, M.W. J. Gen. Virol. 71, 2099-2105 (1990).

9. Jiang, Z., Gera, L., Hartsock, W.J., Hirsch, B., Mant, C.T., Qian, Z., Holmes, K.V., and Hodges, R.S. In Peptides: Peptides Across the Pacific (Proceedings of the 23rd American Peptide Symposium and the 6th International Peptide Symposium), (Lebl, M. Ed.), American Peptide Society and Prompt Scientific Publishing, San Diego, 2013, p. 132-133, http://dx.doi.org/10.17952/23APS.2013/132

10. Lairmore, M.D., DiGeorge, A.M., Conrad, S.F., Trevino, A.V., Lal, R.B., Kaumaya, P.T.P. J. Virol. 69, $6077-$ 6089 (1995).

11. Tripet, B., Kao, D., Jeffers, S., Holmes, K.V., Hodges, R.S. J. Struct. Biol. 155, 176-194 (2006), http://dx.doi.org/10.1016/i.jsb.2006.03.019

12. Yan Z., Hartsock W.J., Qian Z., Holmes K.V., and Hodges R.S., In Small Wonders: Peptides for Disease Control. (Rajasekaran, K., et al. Eds.) American Chemical Society, Washington, DC, 2012, pp. 93-136, http://dx.doi.org/10.1021/bk-2012-1095.ch006 